\title{
Vector Analysis of Changes in the Higher Order Ocular Aberrations and Central Corneal Thickness After T-PRK and Fs-LASIK
}

\author{
Alma Biscevic ${ }^{1,2}$, Ajla Pidro², \\ Melisa Ahmedbegovic- \\ Pjano², Nita Bjedic $^{2}$, Maja \\ Bohac $^{1}$, Sudi Patel ${ }^{3}$ \\ 11University Eye Hospital "Svjetlost" Zagreb. \\ School of Medicine University of Rijeka, Croatia \\ "Eye Clinic "Svjetlost", Sarajevo, Bosnia and \\ Herzegovina \\ ${ }^{3}$ NHS National Services Scotland, Edinburgh, \\ United Kingdom
}

Correspodent author: Alma Biscevic MD, PhD. University Eye Hospital Svjetlost Zagreb, School of Medicine University of Rijeka, Croatia. Address: Heinzelova 39, 10000 Zagreb. E-mail: alma.biscevic@svjetlost.hr..ORCID ID:http// www.orcid.org/: 0000-0002-6496-2853.

doi: 10.5455/aim.2019.28.24-28

ACTA INFORM MED. 2020 MAR; 28(1): 24-28

Received: Jan 09, 2020

Accepted: Feb 16, 2020
(C) 2020 Alma Biscevic, Ajla Pidro, Melisa Ahmedbegovic-Pjano, Nita Bjedic, Maja Bohac, Sudi Patel

This is an Open Access article distributed under the terms of the Creative Commons Attribution NonCommercial License (http://creativecommons.org/ licenses/by-nc/4.0/) which permits unrestricted noncommercial use, distribution, and reproduction in any medium, provided the original work is properly cited.

\begin{abstract}
Introduction: Refractive surgery procedures, transepithelial photorefractive keratectomy (T-PRK) and femtosecond laser in situ keratomileusis (Fs-LASIK) are regarded as safe and efficacious methods for correcting myopia and myopic astigmatism. These two methods do not have much differences in results when treating spherical myopia, while some differences does exist in treatment of myopic astigmatism. Vector analysis presents powerful tool to show the real differences between these two methods regarding higher order ocular aberrations and central corneal thickness of treated eyes. Aim: The aim of the study is to investigate changes in higher order ocular aberrations (HOAs) and central corneal thickness (CCT) following treatment of myopia and myopic astigmatism above -5.00DS and up to -2.00DC after either T-PRK or Fs-LASIK. Methods: Patients (30 eyes per group) underwent T-PRK (group I) or Fs-LASIK (group II) procedure using Schwind Amaris $750 \mathrm{~S}$ laser. HOAs (3mm\&5mm pupil) and CCT were measured objectively at pre-, 1,3 \& 6 months postop in each case. Results: Key results at 6 months were: i) mean values of trefoil ( $5 \mathrm{~mm}$ pupil) were $0.092 \mu \mathrm{m}$ (sd,0.055,95\% Cl 0.072 to 0.112$)$ \& $0.126 \mu \mathrm{m}$ (sd,0.078,95\% Cl 0.098 to 0.154 ) in group I, and $0.088 \mu \mathrm{m}$ (sd, $0.058,95 \% \mathrm{Cl} 0.067$ to 0.109 ) \& $0.064 \mu \mathrm{m}$ (sd, $0.034,95 \% \mathrm{Cl} 0.052$ to 0.076 ) in group II ( $\mathrm{P}=0.001$ at 6 months); ii) Changes in CCT $(\triangle \mathrm{CCT})$ and best spherical equivalent correction $(\triangle B S E)$ was significant in group II $(\triangle C C T=-26.55[\triangle B S E]-14.06, R=0.486, P=0.006)$ but not in group I $(p=0.034)$. Conclusions: After T-PRK trefoil is worse than Fs-LASIK. The predictability of corneal changes is better following Fs-LASIK. .
\end{abstract}

Keywords: Higher order ocular aberrations, Central corneal thickness, T-PRK, FsLasik.

\section{INTRODUCTION}

Transepithelial photorefractive keratectomy (T-PRK) presents a procedure where the corneal epithelium is removed by photo-ablation. This is regarded as a less painful more efficient procedure compared with the more traditional techniques involving either manual scraping or alcohol rub associated with PRK (1-3, 4-9). The change in central corneal thickness (CCT) following myopic corrections by PRK tend to be less compared with LASIK $(10,11)$. Thus, on a likefor-like basis, T-PRK is expected to have less of an impact on CCT than LASIK. However, increases in higher order aberrations (HOAs) such as spherical aberration, coma and trefoil have been reported after the T-PRK procedure $(5,11$, 12). Femtosecond assisted laser in situ keratomileusis (Fs-LASIK) has been shown to be more predictable compared with LASIK (6). The efficacy and safety of both Fs-LASIK and T-PRK procedures has been reported by many groups. However, other than studies by Ghadfan et al. (1) and Luger et al. (2), there is lack of studies directly comparing the outcomes of T-PRK with Fs-LASIK with respect to unexpected higher order aberrations and changes in СCT.

\section{AIM}

The aim of this study was to compare and contrast the changes in 
specific HOAs (namely spherical aberration, coma and trefoil) and CCT after T-PRK and Fs-LASIK procedure.

\section{METHODS}

\section{Patient Population}

This was a retrospective study based on 30 patients that underwent T-PRK and 30 patients that underwent Fs-LASIK. All surgical procedures were performed using Schwind Amaris 750 S excimer laser, for moderate to high myopia or myopic astigmatism patients at University Eye Hospital Svjetlost, Zagreb Croatia, between January 2016 and January 2017. The inclusion criteria were: Preop refractive error between $-5.00 D S$ and -7.00DS with astigmatism no greater than -2.00DC. No previous history of either refractive surgery or anterior segment conditions known to affect the outcome of any corneal refractive procedure. and follow-up time of 6 months with full refractive data available.

Exclusion criteria were thin corneas $(<475 \mu \mathrm{m})$, predicted postop stromal bed thickness less than $280 \mu \mathrm{m}$, any clinical signs of keratoconus, posterior corneal surface elevation of more than $+15.0 \mu m$ evaluated at the thinnest point, estimated postoperative SimK values of less than 35.0D or greater than 49.0D, any ocular opacities that could affect visual acuity or signs of progressive retinal disorders. Patients were offered either the T-PRK or the Fs-LASIK procedure. The pros and cons of both procedures were fully explained to all patients. Patients decided freely on their choice of surgery guided by their personal views or economic factors (T-PRK costs less than Fs-LASIK). Patients with central corneal thickness below 500 microns $(\mu \mathrm{m})$ were advised to opt for T-PRK. Each patient was assigned to a particular treatment group by administrative staff according to the patient's scheduling needs and availability of technical teams required for each procedure. Although this was not a true randomization process, the surgeon performing the treatment had no influence on patient assignment to the groups. The retrospective analysis of the data was approved by the local Ethics committee. All patients gave their informed consent in accordance with the tenets of the Declaration of Helsinki. For patients in whom both eyes fulfilled the defined criteria, only data harvested from right eyes were included in the analytical procedures.

\section{Preoperative Examinations}

Complete preoperative ophthalmological examinations were performed, including corneal topography and pachymetry (Pentacam HRTM, Oculus Optikgeräte $\mathrm{GmbH}$, Wetzlar, Germany) and aberrometry (L8owave+TM, LuneauSAS, Prunay-le-Gillon, France).

Transepithelial photorefractive Keratectomy (TPRK)

For T-PRK, the T-PRK program of the Schwind Amaris $750 \mathrm{OS}$ (Schwind eye-tech-solutions, Kleinostheim,

Germany) was used in all cases. Each treatment was planned before surgery using a Sirius planning system and transferred to an SD card that was inserted into the laser. Epithelial removal (50 microns of epithelium selected automatically by the manufacturer) and excimer laser ablation was performed in one step in all cases.

Femtosecond-Assisted Laser in Situ Keratomileusis (Fs-LASIK)

The procedure included flap of $90 \mu \mathrm{m}$ preparation using an Intralase iFS (150 Hz) femtosecond laser (Abbot Medical Optics, Inc.) which creates an expanding bubble layer of $\mathrm{CO} 2$ and water. The laser was directed at $90 \mu \mathrm{m}$ of corneal thickness in a spiral and raster pattern to achieve planar corneal flap. Focal point was $1 \mathrm{~mm}$ of range which expanded to $2-3 \mathrm{~mm}$ after the photo-disruptive procedure that separated the corneal layers. During the entire procedure, suction was applied and ceased when after the flap was created.

\section{Excimer Ablation}

In all cases, the photoablation was performed with the Schwind Amaris $750 \mathrm{~S}$ excimer laser platform (Schwind eye-tech-solutions, Kleinostheim, Germany) under constant eye tracking conditions. This laser platform features a five-dimensional $1050 \mathrm{~Hz}$ infrared eye tracker with simultaneous limbus, pupil, iris recognition, and cyclotorsion tracking integrated in the laser delivery process. The optical zone was selected depending on the individual mesopic pupil diameter (range 6.5 to $7.0 \mathrm{~mm}$ ) but did not exceed $9.0 \mathrm{~mm}$ zone of total ablation. The Aberration FreeTM program was used in all cases.

\section{Postoperative examinations}

All eyes were checked at the slit lamp at all postop visits. Patients receiving Fs-LASIK were examined after 1 day and 1 week, and those receiving T- PRK after 1,4 days (depending on the epithelial healing and when the bandage contact lens was removed) and 1 week. Thereafter, follow-up visits were scheduled for 1,3 , and 6 months.

\section{Data and Statistical Analysis}

Data were analyzed to determine the significance of changes in the higher order aberrations (HOAs) and central corneal thickness (CCT), within each group and between groups. The t-test, 2 sample assuming unequal variances, was applied when data were normally distributed. Appropriate non-parametric tests were applied when data were not normally distributed. Changes and differences were considered statistically significant when $\mathrm{P}<0.05$. The data were further analyzed as follows: a) to determine the significance of any difference in the mean HOA values between the two groups both before and after treatment (t test); b) to determine the correlation between the change $(\Delta)$ in each HOA value and pretreatment HOA value within each of the two groups (Pearson correlation); c) to determine the significance of any difference in the mean CCT values between the two groups both before and after treatment ( $\mathrm{t}$ test); d) to determine the correlation between the change $(\Delta)$ in each CCT value and difference $(\Delta)$ between best spherical equivalent correction (BSE) at preop and BSE at postop within each of the two groups (Pearson correlation). The significance level was initially set at $\mathrm{P}<0.05$. This was adjusted to $\mathrm{P}<0.009$ using the Bonferroni correction for the number of parameters under consideration. 


\section{RESULTS}

Thirty patients underwent T-PRK and 30 patients underwent the Fs-LASIK (only right eyes in bilateral cases). No major complications associated with surgery were encountered. This paper is centered on the refractive outcomes as noted from the outset. A summary of the main results of refraction are shown in Table 1.

\subsection{High order aberrations}

Chief results are shown in Table 1 and Figure 1. In the T-PRK group for the $3 \mathrm{~mm}$ pupil coma increased by a significant amount $(\mathrm{P}=0.001)$ from a mean value of $0.053 \mu \mathrm{m}$ (sd $\pm 0.024,95 \%$ CI 0.044 to 0.062 ) to $0.098 \mu \mathrm{m}$ ( $\mathrm{sd} \pm 0.069,95 \%$ CI 0.073 to 0.123 ) by 1 month postop. There were no other significant differences between preand postop values of coma for $3 \mathrm{~mm}$ or $5 \mathrm{~mm}$ pupils. The mean value of trefoil increased significantly at 1 month postop by a significant amount $(\mathrm{P}<0.001)$ for the $3 \mathrm{~mm}$ pupil from $0.046 \mu \mathrm{m}$ (sd \pm 0.022 , 95\% CI 0.038 to 0.054 ) to $0.122 \mu \mathrm{m}$ ( $\mathrm{sd} \pm 0.089,95 \%$ CI 0.091 to 0.154 ). There were no other significant differences between pre- and postop values in trefoil for the $3 \mathrm{~mm}$ pupil. For the $5 \mathrm{~mm}$ pupil the mean value of trefoil increased significantly $(\mathrm{P}<0.001)$ from $0.092 \mu \mathrm{m}$ (sd $0.055,95 \%$ CI 0.072 to 0.112 ) to $0.222 \mu \mathrm{m}$ (sd $\pm 0.119,95 \%$ CI 0.179 to 0.265 ) and $0.185 \mu \mathrm{m}$ (sd \pm 0.107 , 95\% CI 0.147 to 0.223 ) at 1 and 3 months postop. The significance of these changes was nullified by 6 months postop. For the $3 \mathrm{~mm}$ pupil spherical aberration significantly changed $(\mathrm{P}<0.001)$ from a mean of $0.003 \mu \mathrm{m}$ (sd $\pm 0.018,95 \%$ CI -0.003 to 0.009 ) to $-0.030 \mu \mathrm{m}$ (sd \pm 0.042 , $95 \%$ CI -0.045 to -0.015 ) and $-0.017 \mu \mathrm{m}$ (sd 0.024, 95\% CI -0.026 to -0.008$)$ at 1 and 3 months postop. The significance of these changes was nullified by 6 months postop. Changes in the mean spherical aberration for the $5 \mathrm{~mm}$ pupil were insignificant. In the Fs-LASIK group there were no significant changes in mean values of either trefoil or spherical aberration. For the $5 \mathrm{~mm}$ pupil the mean value of coma increased significantly $(\mathrm{P}<0.001)$ from $0.102 \mu \mathrm{m}$ ( $\mathrm{sd} \pm 0.038,95 \%$ CI 0.088 to 0.116 ) to $0.197 \mu \mathrm{m}$ (sd $\pm 0.092,95 \% \mathrm{CI} 0.164$ to 0.230 ) and $0.175 \mu \mathrm{m}$ (sd $\pm 0.107,95 \%$ CI 0.137 to 0.213 ) at 1 and 3 months postop. The significance of these changes was

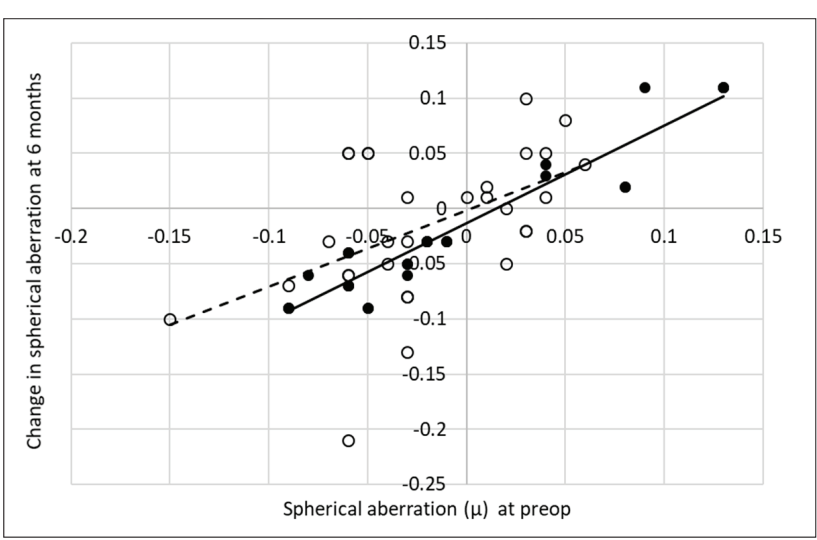

Figure 1. Change in spherical aberration compared and preop value over $5 \mathrm{~mm}$ pupil.

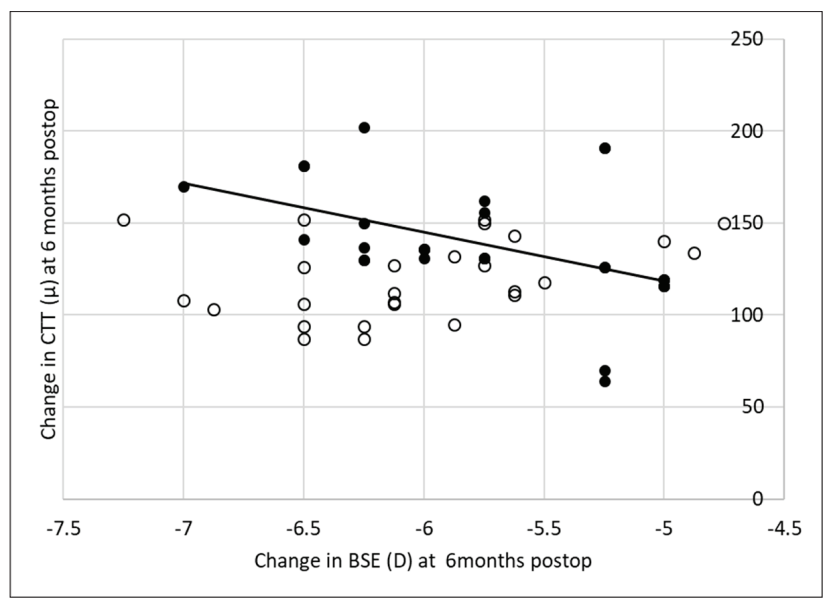

Figure 2. Changes in central corneal thickness and corresponding best spherical equivalent correction at six months postop.

nullified by 6 months postop. There were no significant changes in mean values of coma for the $3 \mathrm{~mm}$ pupil.

Intergroup comparison of the HOA values at each time point

There were significant intergroup differences in coma for the $3 \mathrm{~mm}$ pupil at pre- and 1 month $(\mathrm{P}<0.001)$ but not at 3 and 6 months postop. For coma over the $5 \mathrm{~mm}$ pupil there were no incidences of significant differences between the groups. With regard to trefoil, at preop differences between the two groups were insignificant but significant differences were observed at 1 , ( $3 \mathrm{~mm}$ and $5 \mathrm{~mm}$ pupils, $\mathrm{P}<0.001), 3$ ( $5 \mathrm{~mm}$ pupil $\mathrm{P}<0.001$ ) 

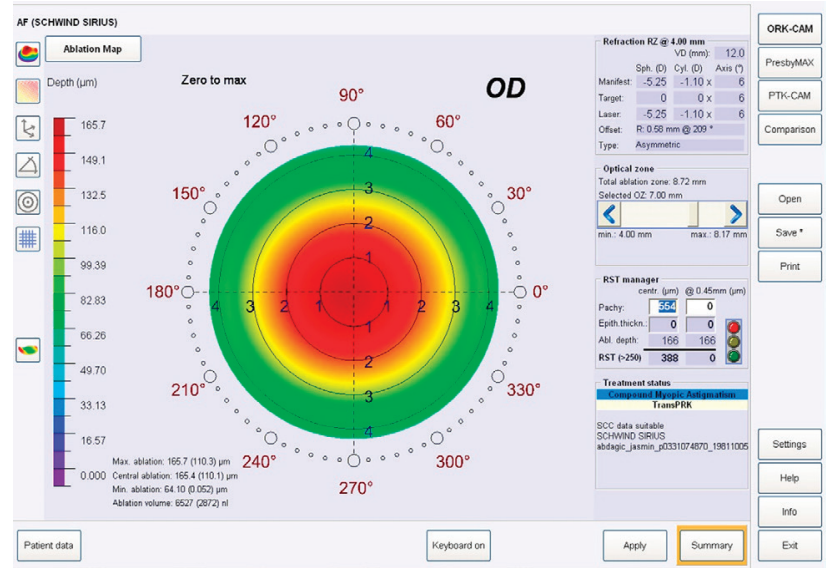

Figure 3. Sirius diagnostic machine display of planned myopic astigmatism treatment with T-PRK method

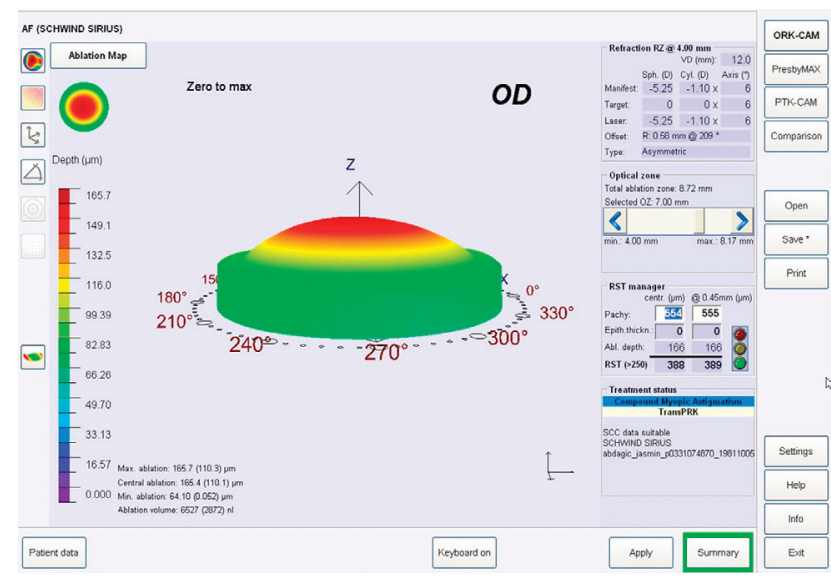

Figure 4. Treatment plan of excimer laser ablation with T-PRK method

and 6 months (3mm and 5mm pupils, $\mathrm{P}<0.001)$. Turning to spherical aberration (SA), at preop differences between the two groups were insignificant but significant differences were observed at $1(5 \mathrm{~mm}$ pupils $\mathrm{P}=0.003)$ and 3 ( $3 \mathrm{~mm}$ and $5 \mathrm{~mm}$ pupils, $\mathrm{P}=0.004$ ) months postop but not at 6 months.

Correlation between $\triangle \mathrm{HOA}$ and preop HOA values within each group

In the T-PRK group there were no significant associations between $\Delta$ coma and preop coma or $\Delta$ trefoil

and preop trefoil for both $3 \mathrm{~mm}$ and $5 \mathrm{~mm}$ pupils. A significant association between $\Delta$ spherical aberration $(\triangle \mathrm{SA})$ and preop spherical aberration (SA) was found at 6 months postop for the $5 \mathrm{~mm}$ pupil. The least squares regression lines equating $\triangle \mathrm{SA}$ and $\mathrm{SA}$ was as follows:

$\triangle \mathrm{SA}=0.689 \mathrm{SA}-0.002(\mathrm{R}=0.499, \mathrm{~N}=30, \mathrm{P}=0.005)$ eq. 1

In the Fs-LASIK group, significant associations were revealed between, $\Delta$ coma and preop coma for the $3 \mathrm{~mm}$ pupil only at all time postop periods, $\Delta$ trefoil and preop trefoil for both pupil sizes at all three postop periods, $\triangle$ SA and preop SA for both pupil sizes at 3 and 6 months postop. The least squares regression line equating $\triangle \mathrm{SA}$ and SA at 6 months postop for the $5 \mathrm{~mm}$ pupil was as follows:

$\Delta \mathrm{SA}=0.884 \mathrm{SA}-0.014(\mathrm{R}=0.952, \mathrm{~N}=30, \mathrm{P}<0.001)$ eq. 2 .

The difference between the two matched correlation coefficients was significant $(Z=-4.781, P<0.001)$. Figure 1 show the relationships between the change in spher-

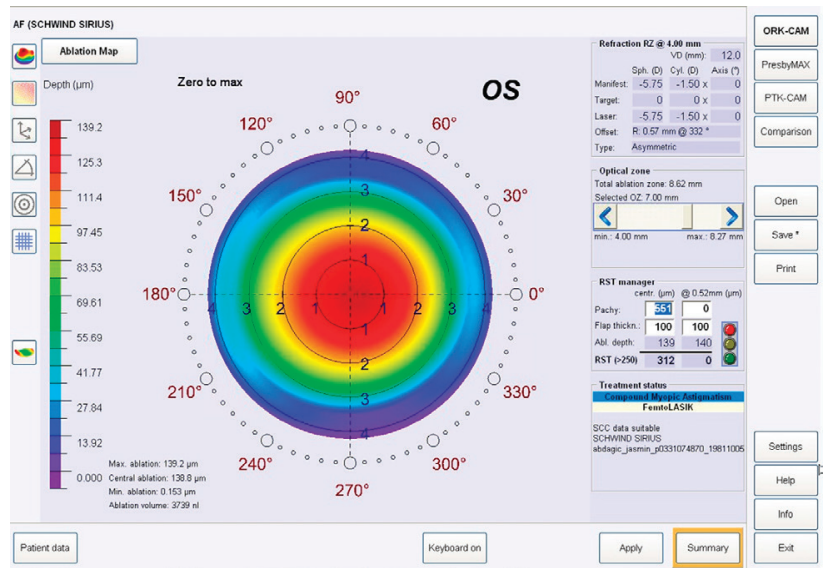

Figure 5. Sirius diagnostic machine display of planned myopic astigmatism treatment with Fs-LASIK method

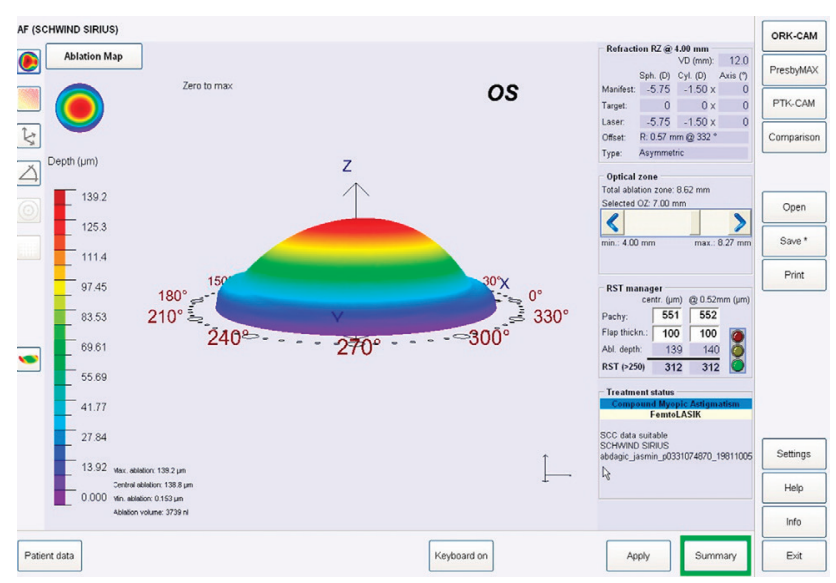

Figure 6. Treatment plan of excimer laser ablation with Fs-LASIK method

ical aberration observed at 6 months postop and the respective preop values in each case.

\subsection{Pachymetry}

Chief results are shown in Figure 2. In the T-PRK group mean central corneal thickness (CCT) was

$512.4 \mu \mathrm{m}$ (sd $\pm 23.7,95 \%$ CI 504.0 to 520.9 ) at preop and $390.9 \mu \mathrm{m}$ (sd $\pm 32.2,95 \%$ CI 379.4 , to 402.4 ) at 6 months postop. In the Fs-LASIK group, CCT was 551.5 $\mu \mathrm{m}(\mathrm{sd} \pm 21.7,95 \%$ CI 504.0 to 520.8$)$ at preop and 413.3 $\mu \mathrm{m}(\mathrm{sd} \pm 30.2,95 \% \mathrm{CI} 379.4$ to 402.4$)$ at 6 months postop. Intergroup differences were significant on all occasions $(\mathrm{P}<0.001)$.

Correlation between $\triangle C C T$ and preop $\triangle B S E$ values

At 6 months postop the mean change in best spherical equivalent (BSE) was $-5.84 \mathrm{DS}$ (sd $\pm 0.84,95 \% \mathrm{CI}$

-6.14 to -5.54$)$ in the T-PRK group and $-5.91 \mathrm{DS}$ $(\mathrm{sd} \pm 0.60,95 \% \mathrm{CI}-6.12$ to -5.70$)$ in the Fs-LASIK group. The difference between the groups was not significant. In the T-PRK group there was no significant association between $\triangle C C T$ and $\triangle B S E$ ( $p=0.034$ ) after the Bonferroni correction. In the Fs-LASIK group there was a significant association between $\triangle \mathrm{CCT}$ and $\triangle \mathrm{BSE}$ where:

$\Delta \mathrm{CCT}=-26.55(\Delta \mathrm{BSE})-14.06(\mathrm{R}=0.486, \mathrm{~N}=30, \mathrm{P}=0.006)$ eq.3.

\section{DISCUSSION}

Table 1 shows, the mean values for coma, trefoil and spherical aberration tended to increase after T-PRK. 
These results confirm previous reports $(1,4,12,13)$ However, by 6 months postop the changes we observed were not significant when compared with preop values. In the Fs-LASIK group, mean values for the HOAs remained moderately stable throughout, except for coma that tended to increase but reverted back towards the preop level by 6 months postop. At preop, there were no significant intergroup differences for trefoil and spherical aberration. However, at various points over the postop period significant intergroup differences were exposed. The indices of the best fit equations verify that, on an individual case-by case basis, changes in the magnitude of HOAs after Fs-LASIK are highly predictable. This cannot be said for T-PRK except for changes in spherical aberration at 6 months postop (eq.1). Though changes in spherical aberration can be predicted in T-PRK cases, the differences between eqs 1 and 2 reveal that predicting individual changes after Fs-LASIK are more precise, less prone to error, than T-PRK. T-PRK tends to have a more adverse effect on HOAs compared with Fs-LASIK. Wen et al. (6) concluded there was no real difference in the HOAs between T-PRK and Fs-LASIK.

Our findings are not in accordance with their conclusion. Over the period of investigation, the mean central corneal thickness (CCT) was always greater in the Fs-LASIK group. Extrapolating from our results, the change in CCT is $20.9 \mu$ per diopter of BSE after T-PRK and $23.4 \mu$ per diopter of BSE after Fs-LASIK. This is a clear indication of the tissue saving advantage of T-PRK over Fs-LASIK. Kriegerowski et al. (14) showed that changes in the structure of the corneal stroma along the antero-posterior depth of the stroma affects the rate of photoablation at different regions of the stroma. The anterior stroma is more amenable to photoablation and, as such, less tissue is required to be removed from the anterior region in order to achieve a change in unit change in refraction. Our results comply with this assertion.

The relationship between $\triangle \mathrm{CCT}$ and $\triangle \mathrm{BSE}$, as shown in Figure 2, for the T-PRK cases $(\mathrm{P}=0.034)$ was deemed insignificant after making the Bonferroni correction but this was not the case after Fs-LASIK. The indices of eq.3 signify that estimating changes in CCT after Fs-LASIK for myopia and/or myopic astigmatism is highly predictable. A feature that cannot be said for T-PRK. In keeping with more traditional findings after PRK, Hou et al. (15) found central epithelial thickness increased between 1 and 6 months after T-PRK. In addition, they found greater amounts of thickening took place in paracentral regions of the cornea resulting in an epithelial lenticule with the appearance of a negatively powered lens. Modulation of epithelial thickness distribution could be the source of uncertainty in the $\triangle \mathrm{CCT}$ and $\triangle \mathrm{BSE}$ relationship after T-PRK.

\section{CONCLUSION}

Both corneal surgical methods for treating myopia and myopic astigmatism show safe and efficient results when dealing with higher order ocular aberrations and central corneal thickness. However, after T-PRK trefoil is worse than Fs-LASIK. The predictability of corneal changes is better following Fs-LASIK.

Author's contribution: A.B, A.P. and M.A.P gave substantial contributions to the conception or design of the work in acquisition, analysis, or interpretation of data for the work. N.B., M.B and S.P had a part in article preparing for drafting or revising it critically for important intellectual content, and A.B and M.B gave final approval of the version to be published and agreed to be accountable for all aspects of the work.

- Conflicts of interest: There are no conflicts of interest.

Financial support and sponsorship: Nil.

\section{REFERENCES}

1. Ghadhfan F, Al-Rajhi A, Wagoner MD. Laser in situ keratomileusis versus surface ablation: visual outcomes and complications. J Cataract Refract Surg. 2007; 33: 2041-2048.

2. Luger MH, Ewering T, Arba-Mosquera S. Myopia correction with transepithelial photorefractive keratectomy versus femtosecond assisted laser in situ keratomileusis: one-year case-matched analysis. J Cataract Refract Surg 2016; 42: 1579-1587.

3. Aslanides IM, Georgoudis PN, Selimis VD, Mukherjee AN. Single-step transepithelial ASLA (SCHWIND) with mitomycin-C for correction of high myopia: long term follow-up. Clin Ophtalmol. 2015; 9: 33-41.

4. Adib-Moghaddam S, Soleyman-Jahi S, Salmanian B. et al. Single-step transepithelial photorefractive keratectomy in myopia and astigmatism: 18-month follow-up. J Cataract Refract Surg. 2016; 42: 1570-1578.

5. Antonios R, Abdul Fattah M, Arba Mosquera S. et al. Single-step transepithelial versus alcohol-assisted photorefractive keratectomy in the treatment of high myopia: a comparative evaluation over 12 months. $\mathrm{Br}$ J Ophthalmol. 2017; 101: 1106-1112.

6. Wen D, McAlinden C, Flitcroft I. et al. Postoperative Efficacy, Predictability, Safety, and Visual Quality of Laser Corneal Refractive Surgery: A Network Meta-analysis. Am J Ophthalmol. 2017; 178: 65-78.

7. Celik U, Bozkurt E, Celik B. et al. Pain, wound healing and refractive comparison of mechanical and transepithelial debridement in photorefractive keratectomy for myopia: results of 1 year follow-up. Cont Lens Anterior Eye. 2014; 37(6): 420-426.

8. Zarei-Ghanavati S, Shandiz JH, Abrishami M, Karimpour M. Comparison of mechanical debridement and trans-epithelial myopic photorefractive keratectomy: A contralateral eye study. J Curr Ophthalmol. 2019; 23; 32: 135-141.

9. Fattah MA, Antonios R, Arba Mosquera S et al. Epithelial Erosions and Refractive Results After Single-Step Transepithelial Photorefractive Keratectomy and Alcohol-Assisted Photorefractive Keratectomy in Myopic Eyes: A Comparative Evaluation Over 12 Months. Cornea. 2018; 37 : 45-52.

10. de Ortueta D, von Rüden D, Verma S. et al. Transepithelial Photorefractive Keratectomy in Moderate to High Astigmatism With a Non-wavefront-Guided Aberration-Neutral Ablation Profile. J Refract Surg. 2018; 34(1): 466-474

11. Pokroy R, Mimouni M, Sela T, Munzer G, Kaiserman I. Predictors of myopic photorefractive keratectomy retreatment. J Cataract Refract Surg. 2017; 43: 825-832.

12. Thibos LN, Wheeler W, Horner D. Power Vectors: an Application of Fourier Analysis to the Description and Statistical Analysis of Refractive Error. Optom Vis Sci. 1997; 74: 367-375.

13. Alpins NA. A New Method of Analyzing Vectors for Changes in Astigmatism. J Cataract Refract Surg. 1993; 19: 524-533.

14. Kriegerowski M, Bende T, Seiler T, et al. The Ablation Behavior of Various Corneal Layers. Fortschr Ophthalmol. 1990;87: 11-13.

15. Hou J, Wang Y, Lei Y, et al. Corneal Epithelial Remodeling and its Effect on Corneal Asphericity after Transepithelial Photorefractive Keratectomy for Myopia. J Ophthalmol. 2016; 85: 823-862. 